\title{
Localization of Parotid Gland Tumors in Relation to the Intraparotid Facial Nerve on 3D Double-Echo Steady-State with Water Excitation Sequence
}

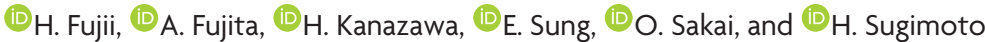

\begin{abstract}
BACKGROUND AND PURPOSE: Reliable preoperative facial nerve mapping may help avoid or minimize facial nerve injury during parotid tumor resection. The purpose of this study was to investigate the diagnostic performance of the 3D double-echo steady-state with water excitation sequence in localizing parotid gland tumors through direct visualization of the intraparotid facial nerve in comparison with indirect methods of estimating the facial nerve location.
\end{abstract}

MATERIALS AND METHODS: We retrospectively reviewed 91 parotid gland tumors in 90 patients who underwent surgical resection and preoperative MR imaging, including the 3D double-echo steady-state with water excitation sequence. The tumor locations were categorized as deep or superficial on the basis of direct and 3 indirect methods: the facial nerve line, retromandibular vein, and Utrecht line. Surgical localization was considered the criterion standard. The diagnostic performance for localizing deep lobe lesions using direct and indirect methods was calculated and compared using the McNemar test.

RESULTS: Surgical localization confirmed 75 superficial lesions and 16 deep lesions. The interobserver variability of the 3D double-echo steady-state with water excitation sequence was excellent $(\kappa=0.870)$. The diagnostic accuracy, sensitivity, specificity, positive predictive value, and negative predictive value for localizing deep lobe lesions using the 3D double-echo steady-state with water excitation method were $97.8 \%, 87.5 \%, 100 \%, 100 \%$, and $97.4 \%$, respectively. These findings were significantly higher than the facial nerve line in sensitivity, the retromandibular vein in sensitivity, and the Utrecht line in accuracy and specificity $(P<.05)$. Overall, the direct method was the most accurate, sensitive, and specific in localizing parotid gland tumors.

CONCLUSIONS: We can achieve higher diagnostic performance in localizing parotid gland tumors by directly visualizing the intraparotid facial nerve using the 3D double-echo steady-state with water excitation sequence compared with indirect methods.

ABBREVIATIONS: 3D-DESS-WE = 3D double-echo steady-state with water excitation; $F N L=$ facial nerve line; $\mathrm{RMV}=$ retromandibular vein; $\mathrm{UL}=\mathrm{Utrecht}$ line

$\mathbf{T}$ he facial nerve exits the skull base via the stylomastoid foramen and enters the parotid gland. Within the parotid gland, the intraparotid facial nerve trunk divides into its 2 main branches, the temporofacial and cervicofacial branches, and fur-

Received December 17, 2018; accepted after revision April 21, 2019.

From the Department of Radiology (H.F., A.F., H.K., H.S.), Jichi Medical University, School of Medicine, Tochigi, Japan; and Departments of Radiology (E.S., O.S.), Otolaryngology-Head and Neck Surgery (O.S.), and Radiation Oncology (O.S.), Boston Medical Center, Boston University School of Medicine, Boston, Massachusetts.

Paper previously presented, in part, at: Scientific Assembly and Annual Meeting of the Radiological Society of North America, November 27 to December 2, 2016; Chicago, Illinois (Hiroyuki Fujii, Akifumi Fujita, Hidenori Kanazawa, Edward Sung, Osamu Sakai, Hideharu Sugimoto. "Localization of Parotid Gland Tumors in Relation to the Intraparotid Facial Nerve on 3D-Double-Echo Steady-State with Water Excitation Sequence").

Please address correspondence to Hiroyuki Fujii, MD, PhD, Department of Radiology, Jichi Medical University School of Medicine, 3311-1 Yakushiji, Shimotsuke,

Tochigi 329-0498, Japan; e-mail: hiroyuki.fuji@jichi.ac.jp

http://dx.doi.org/10.3174/ajnr.A6078 ther divides into the temporal, zygomatic, buccal, mandibular, and cervical branches. The intraparotid facial nerve and its branches course predominantly in a single sagittal plane, which separates the superficial and deep lobes of the parotid gland. ${ }^{1}$ It is important to know the spatial relationship of the intraparotid facial nerve to a parotid tumor because this information may impact the duration and difficulty of the operation. ${ }^{2}$ Several indirect methods to estimate the location of the intraparotid facial nerve have been reported. ${ }^{3-6}$ However, the accuracy of indirect methods is variable and can be affected by tumor size, location, and the specific method used. Therefore, direct visualization of the intraparotid facial nerve is desirable. ${ }^{7}$ With increasing spatial and contrast resolution of cross-sectional imaging, better visualization of the cranial nerves has become possible. ${ }^{2,8,9}$ However, imaging the course of the intraparotid facial nerve still remains a diagnostic challenge due to the fine structure and complex anatomy of the nerve. The 3D double-echo steady-state with water 
excitation (3D-DESS-WE) sequence is a recently introduced MR imaging technique that can delineate the peripheral cranial nerves as high-signal-intensity structures. ${ }^{10,11}$ At Jichi Medical University Hospital, this sequence has been added to our standard MR imaging protocol of the salivary glands and has been used routinely to demonstrate the intraparotid facial nerve and salivary ducts within the salivary glands since 2012. The purpose of this study was to investigate the accuracy of the 3D-DESS-WE MR imaging sequence in localizing parotid gland tumors through direct visualization of the intraparotid facial nerve and to compare it with indirect methods of localization.

\section{MATERIALS AND METHODS \\ Patient Population}

This retrospective study was approved by our institutional review board, and informed consent was waived. We retrospectively reviewed 107 consecutive patients (53 men and 54 women with a median age of 58 years; range, $19-87$ years) with parotid gland lesions who underwent surgical resection and preoperative MR imaging, including the 3D-DESS-WE sequence from October 2012 to March 2018. Two patients with severe motion artifacts, and 15 patients who could not have accurate surgical confirmation of parotid tumor localization (enucleation, $n=10$, and total parotidectomy, $n=5$ ) were excluded. Ultimately, 91 parotid gland tumors in 90 patients were included in this study (1 patient had ipsilateral multiple parotid gland tumors).

\section{MR Imaging and Interpretation}

All patients underwent MR imaging with a 3T unit (Magnetom Skyra; Siemens, Erlangen, Germany) using a commercially available 20-channel head and neck coil with our routine neck protocol that included the 3D-DESS-WE sequence. The parameters of the 3D-DESS-WE sequence are as follows: TR/TE $=11 / 4.21 \mathrm{~ms}$, flip angle $=30^{\circ}, \mathrm{FOV}=200 \times 200 \mathrm{~mm}$, matrix $=384 \times 244$, effective section thickness $=0.82 \mathrm{~mm}$, number of acquisitions $=1$, scan time $=4$ minutes 12 seconds. The slab thickness $(11.2 \mathrm{~cm})$ of the 3D-DESS-WE sequence is from the skull base to the mandible.

All acquired images were transferred to our clinically used server and evaluated with a PACS system with a 2-megapixel high-resolution liquid crystal display. Two board-certified radiologists (with 21 and 7 years of post training experience in head and neck radiology, respectively) independently evaluated the location of the parotid gland lesions in preoperative MR imaging. The locations of the parotid gland lesions were categorized into the superficial or deep lobe based on direct and 3 indirect methods.

\section{Direct Method with Visualization of the Intraparotid Facial Nerve on 3D-DESS-WE}

The intraparotid facial nerve was identified on the 3D-DESS-WE sequence (Fig 1) using axial source images and multiplanar reconstructions, which were created by radiology technologists. Parotid tumors lateral to the intraparotid facial nerve were defined as being in the superficial lobe (Fig 2), and parotid tumors medial to the intraparotid facial nerve were defined as being in the deep lobe (Fig 3). When peripheral facial nerve branches could not be delineated to determine the tumor location, a plane parallel to the

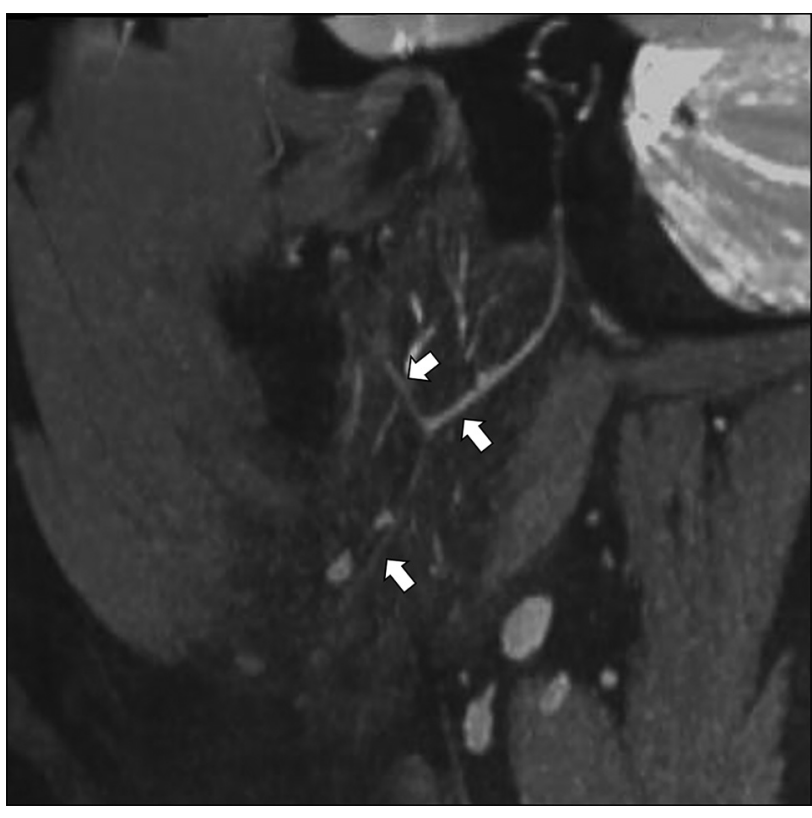

FIG 1. Visualization of the intraparotid facial nerve on the 3DDESS-WE sequence. Reformatted sagittal image of the 3D-DESS-WE sequence clearly shows the intraparotid facial nerve (arrows).

visualized main trunk of the intraparotid facial nerve was used to localize the parotid gland tumor, on the basis of the assumption that the deviation of the facial nerve trunk could determine the tumor location. Tumors totally or with $>50 \%$ of tumor volume located lateral to the plane were defined as being in the superficial lobe, and tumors totally or with $>50 \%$ of tumor volume located medial to the plane were defined as being in the deep lobe.

\section{Indirect Approximation of the Intraparotid Facial Nerve Using the Facial Nerve Line, Retromandibular Vein, and Utrecht Line}

The 3 indirect methods were evaluated on axial T2-weighted images (TR/TE, 5000/83 ms) and 3D-T1 volumetric interpolated brain examinations with 2-point Dixon technique images (TR/TE, 6/2.46 ms) after intravenous administration of 0.1 $\mathrm{mmol} / \mathrm{kg}$ body weight of a gadolinium contrast agent (Fig 4). The facial nerve line (FNL) connects the lateral surface of the posterior belly of the digastric muscle to the lateral surface of the ascending ramus of the mandible. The Utrecht line (UL) connects the most dorsal point observed on the ipsilateral half of a vertebra to the most dorsal point of the retromandibular vein (RMV). Tumors totally or with $>50 \%$ of tumor volume located lateral to the FNL, UL, or RMV were defined as being in the superficial lobe, and tumors totally or with $>50 \%$ of tumor volume located medial to the FNL, UL, or RMV were defined as being in the deep lobe.

\section{Tumor Location Based on Surgical Findings}

The spatial relationship of the intraparotid facial nerve to a parotid tumor was confirmed by reviewing the surgical report, which was considered the criterion standard and was used to determine the diagnostic performance of using the direct and indirect methods described above. 

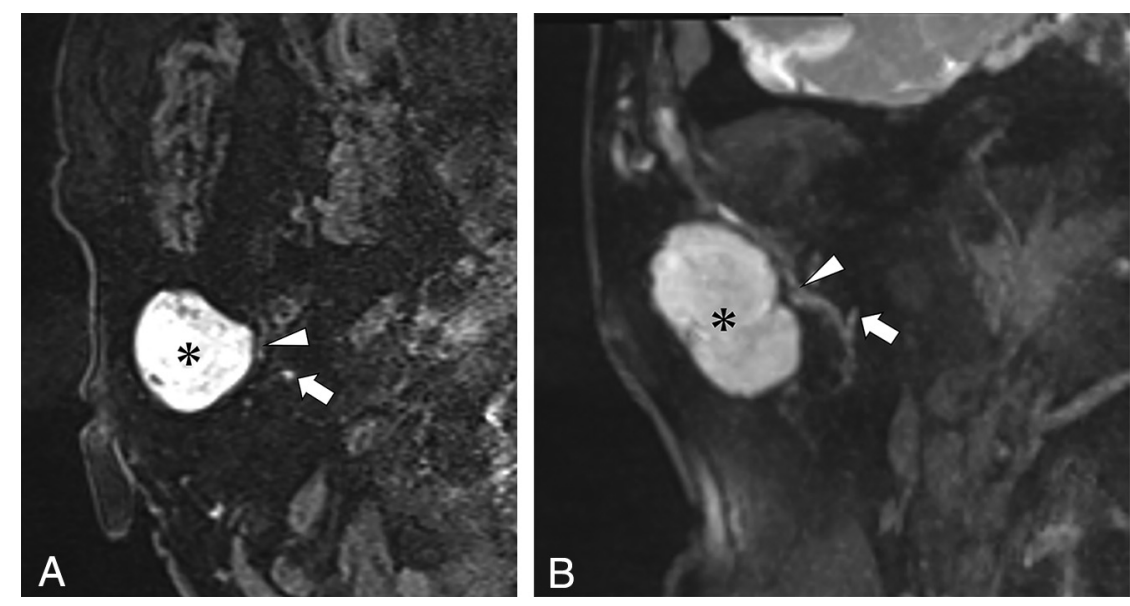

FIG 2. A 71-year-old woman with pleomorphic adenoma of the right parotid gland. A, Axial source image of the 3D-DESS-WE sequence. $B$, Coronal reformatted image of the 3D-DESS-WE sequence. Axial and reformatted coronal images of the 3D-DESS-WE sequence show the intraparotid facial nerve trunk ( $A$ and $B$, arrows) and temporofacial trunk ( $A$ and $B$, arrowheads) coursing medially to the parotid mass ( $A$ and $B$, asterisk), suggesting localization to the superficial lobe.
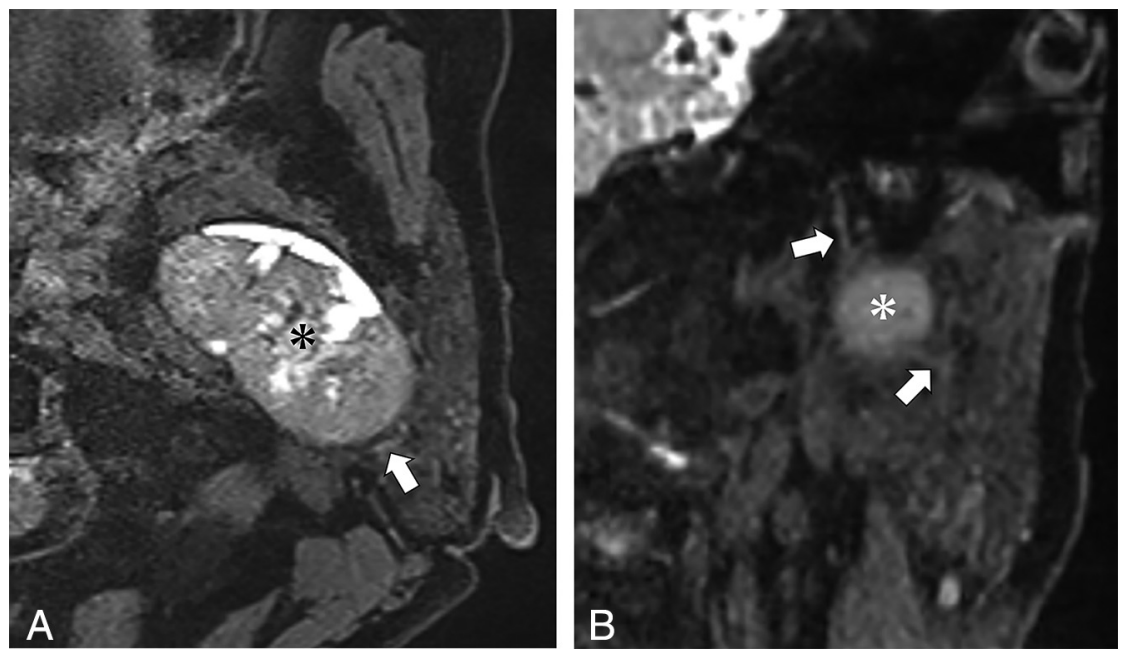

FIG 3. A 65-year-old woman with basal cell adenoma of the left parotid gland. A, Axial source image of the 3D-DESS-WE sequence. $B$, Coronal reformatted image of the 3D-DESS-WE sequence. Axial and reformatted coronal 3D-DESS-WE images show the intraparotid facial nerve trunk (arrows) coursing lateral to the parotid mass (asterisk), suggesting localization to the deep lobe.

\section{Statistical Analysis}

Two readers independently evaluated the tumor locations using the direct and indirect methods. Inconsistencies were resolved by collaborative review and consensus agreement. The interobserver variability was calculated using $\kappa$ analysis: poor, $\kappa \leq 0.2$; fair, $0.2<\kappa \leq 0.4$; moderate, $0.4<\kappa \leq 0.6$; good, $0.6<\kappa \leq 0.8$; excellent, $0.8<\kappa \leq 1$. The diagnostic accuracy, sensitivity, specificity, positive predictive value, and negative predictive value for localizing parotid lesions using direct and indirect methods were calculated and compared using the McNemar test. Given the bivariate (superficial-versus-deep) nature of our data, the sensitivity and specificity and the positive and negative predictive values for superficial and deep lobe analyses were the inverse of each other. Therefore, we chose to focus on the analysis for localizing deep lobe lesions. Statistical analyses were performed using Version 3.3.3 of the R statistical and computing software (http:// www.r-project.org/).

\section{RESULTS}

Surgical findings confirmed 75 lesions localized to the superficial lobe, and 16 lesions localized to the deep lobe. The histologic types and frequencies of parotid lesions are shown in Table 1.

The interobserver variabilities in determining the location of the parotid lesions using direct and indirect methods are shown in Table 2. The interobserver variability of the direct method was excellent (0.870), that of RMV was good (0.706), and those of FNL and UL were moderate ( 0.587 and 0.471 , respectively).

Of the total 91 parotid lesions, 85 lesions could be localized in relation to the intraparotid facial nerve on the 3DDESS-WE sequence. The remaining 6 lesions were located in the far distal (anterior) part of parotid gland parenchyma, rendering visualization of the intraparotid facial nerve limited on the 3D-DESS-WE sequence. In these cases, we categorized the lesion location using a plane parallel to the visualized main trunk of the intraparotid facial nerve (described in the Materials and Methods section).

The diagnostic accuracy, sensitivity, specificity, positive predictive value, and negative predictive value for surgically confirmed deep lobe lesions using direct and indirect methods are shown in Table 3. Of the 4 methods, the direct method showed the highest accuracy, sensitivity, and negative predictive value for localization of deep lobe parotid gland tumors $(97.8 \%, 87.5 \%$, and $97.4 \%$, respectively). The direct method also showed high specificity and positive predictive value, which were identical to those of the RMV (100\% and 100\%, respectively). The accuracy and specificity of the direct method were statistically higher than those of the $\mathrm{UL}(P=.002$ and $P=.008$, respectively). The sensitivity of the direct method was statistically higher than that of the FNL and $\operatorname{RMV}(P=.008$ and $P=.031$, respectively). Overall, the direct method was the most accurate, sensitive, and specific in localizing parotid gland tumors. Among the indirect methods, the RMV showed the highest accuracy, specificity, positive predictive value, and negative predictive value $(91.2 \%, 100 \%, 100 \%, 90.4 \%$, respectively). The FNL showed relatively high specificity but low sensitivity ( $93.3 \%$ and $37.5 \%$, respectively). The UL showed the lowest diagnostic performance among the 4 methods.

\section{DISCUSSION}

Surgery for parotid gland tumors has a relatively high rate of complications, including facial nerve paralysis, salivary fistula, infec- 


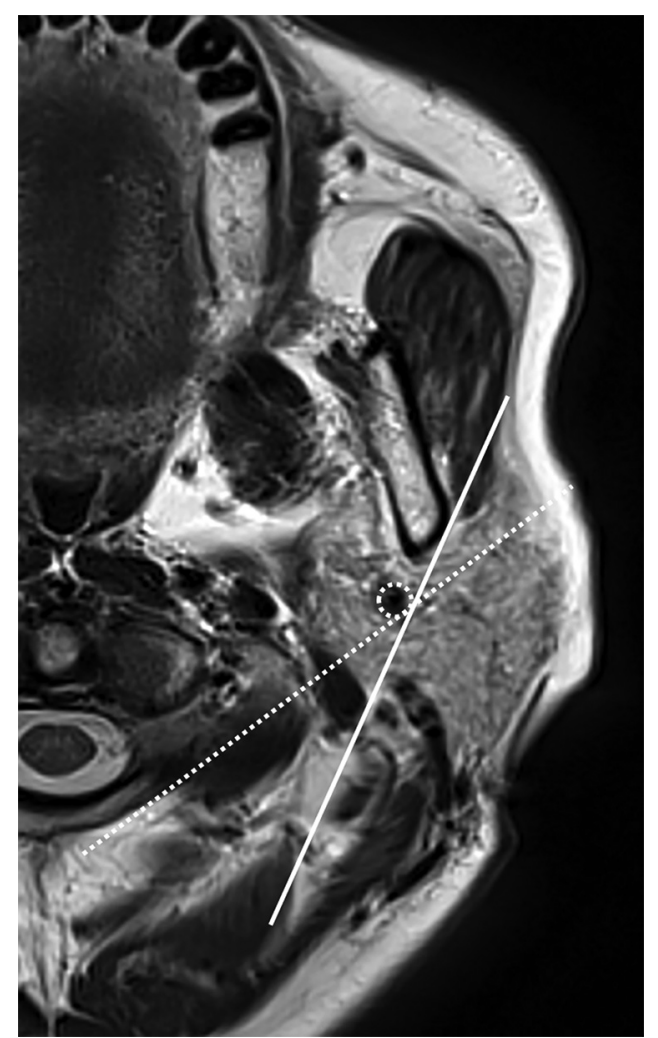

FIG 4. Indirect methods to approximate the intraparotid facial nerve: facial nerve line (solid line), retromandibular vein (dotted circle), and Utrecht line (dotted line).

Table 1: Histologic types and frequencies of parotid lesions

\begin{tabular}{lrcc}
\multicolumn{1}{c}{ Histologic Types } & \multicolumn{3}{c}{ Surgical Findings } \\
\cline { 3 - 5 } & & $\begin{array}{c}\text { Superficial } \\
\text { Lobe }\end{array}$ & $\begin{array}{c}\text { Deep } \\
\text { Lobe }\end{array}$ \\
\hline Benign tumors & 57 & 44 & 13 \\
Pleomorphic adenoma & 15 & 13 & 2 \\
Warthin tumor & 3 & 2 & 1 \\
Basal cell adenoma & 1 & 1 & 0 \\
Myoepithelioma & 3 & 3 & 0 \\
Lymphoepithelial cyst & 1 & 1 & 0 \\
Reactive lymphadenopathy & 1 & 1 & 0 \\
Inflammation & & & \\
Malignant tumors & 1 & 1 & 0 \\
Malignant lymphoma & 2 & 2 & 0 \\
Metastatic tumor & 1 & 1 & 0 \\
Oncocytic carcinoma & 2 & 2 & 0 \\
Acinic cell carcinoma & 1 & 1 & 0 \\
Carcinoma ex pleomorphic adenoma & 1 & 1 & 0 \\
Basal cell adenocarcinoma & 2 & 2 & 0 \\
Salivary duct carcinoma & 91 & 75 & 16 \\
Total & & &
\end{tabular}

Table 2: Interobserver variabilities in determining the location of the parotid lesion with direct and indirect methods

\begin{tabular}{lccc}
\hline $\begin{array}{l}\text { Interobserver } \\
\text { Variability }\end{array}$ & No. & Concordance & $\boldsymbol{\kappa}$ \\
\hline Direct method & 91 & 88 & 0.870 \\
FNL & & 81 & 0.587 \\
RMV & & 86 & 0.706 \\
UL & 75 & 0.471 \\
\hline
\end{tabular}

tion, hematoma, sialocele, and Frey syndrome. Among these, temporary facial nerve paralysis is most frequent. ${ }^{12}$ Facial nerve injury mechanisms during parotidectomy include nerve dissection, stretching, compression, ligature entrapment, thermal and electrical injuries, and ischemia. ${ }^{13}$ Facial nerve paralysis can cause cosmetic and functional morbidity, ocular complications, diminished quality of life, and medical malpractice litigation. ${ }^{14,15} \mathrm{Re}-$ liable preoperative facial nerve mapping may assist safe and effective tumor resection and provide better information for patients regarding potential risks and morbidity after surgical intervention.

Because it is difficult to visualize the intraprarotid facial nerve by routine MR imaging, various indirect methods (such as FNL, RMV, and UL) for approximating the course of the intraparotid facial nerve to aid in localizing parotid gland tumors have been proposed. Several studies have shown high accuracy of the FNL $(71.4 \%-87.5 \%)$, though there was low sensitivity for deep lobe lesions $(12.5 \%-50.0 \%)$ despite high specificity (98.1\%). The RMV has been reported to show relatively high accuracy (63.5\% 86.4\%) along with high specificity (85.7\%-96.2\%) and variable sensitivity $(29.6 \%-71 \%) .^{5,16,17} \mathrm{Kim}$ et al ${ }^{16}$ reported that the UL showed relatively low accuracy $(74.8 \%)$, low sensitivity (18.5\%), and high specificity (89.4\%). In this study, the FNL showed low sensitivity and high specificity, and the RMV showed high accuracy and specificity, findings consistent with previous reports. The ULs showed relatively high accuracy and specificity, but they were still the lowest in our study. Among indirect methods, the RMV showed the best diagnostic performance in this study. Our study also showed that the interobserver variability of indirect methods was lower than that of the direct method. In particular, the dorsal points of the FNL (posterior belly of the digastric muscle) and UL (most dorsal point observed on the ipsilateral half of a vertebra) can vary depending on the imaging slice. In some cases, the branch of the external carotid artery was misinterpreted as the RMV. These factors may have led to the lower interobserver variability. Overall, the broad variability of the diagnostic performance for the indirect methods from previous studies as well as our own study suggests that indirect methods are not reliable for accurate surgical planning, with a higher risk of surgical complications related to facial nerve injury.

The 3D-DESS-WE sequence is commonly used in musculoskeletal imaging, but it was recently used for visualization of the intraparotid facial nerve and has been quite successful. ${ }^{11}$ The $3 \mathrm{D}$ DESS-WE sequence includes both FISP and reversed fast imaging with steady-state free precession components. The FISP signal intensity provides more anatomic details with tissue contrast dominated by the T1/T2 ratio. ${ }^{11}$ The reversed fast imaging with steady-state free precession signal intensity accentuates the signal intensity of the components with a long T2 signal. ${ }^{18}$ With 3DDESS-WE, the facial nerve and its branches can be easily identified because they demonstrate high signal intensity. ${ }^{11}$ Another study showed that the 3D-DESS-WE sequence demonstrated excellent visualization of the entire course of the mandibular division of the trigeminal nerve in most patients. ${ }^{10}$ Therefore, we routinely use the $3 \mathrm{D}$-DESS-WE sequence to visualize the intraparotid facial nerve for preoperative evaluation. To our knowledge, 
Table 3: Localization of parotid lesions with imaging and surgical findings and the diagnostic performance of the surgically confirmed deep lobe lesions ${ }^{a}$

\begin{tabular}{|c|c|c|c|c|c|c|c|}
\hline & \multicolumn{2}{|c|}{ Surgical Findings } & \multicolumn{5}{|c|}{ Diagnostic Performance (Deep Lobe Lesions) (\%) $(95 \% \mathrm{CI})$} \\
\hline & $\begin{array}{l}\text { Deep } \\
\text { Lobe }\end{array}$ & $\begin{array}{c}\text { Superficial } \\
\text { Lobe }\end{array}$ & Accuracy & Sensitivity & Specificity & PPV & NPV \\
\hline \multicolumn{8}{|l|}{ 1) Direct method } \\
\hline Deep lobe & 14 & 0 & $97.8(91.4-97.8)$ & $87.5(69.2-87.5)$ & $100(96.1-100)$ & $100(79.0-100)$ & $97.4(93.6-97.4)$ \\
\hline Superficial lobe & 2 & 75 & & & & & \\
\hline \multicolumn{8}{|l|}{ 2) FNL } \\
\hline Deep lobe & 6 & 5 & $83.5(76.7-89.7)(P=.065)$ & $37.5(18.0-55.0)\left(P=.008^{b}\right)$ & $93.3(89.2-97.1)(P=.063)$ & $54.4(26.2-80.1)$ & $87.5(83.6-91.0)$ \\
\hline Superficial lobe & 10 & 70 & & & & & \\
\hline \multicolumn{8}{|l|}{ 3) RMV } \\
\hline Deep lobe & 8 & 0 & $91.2(84.7-91.2)(P=.727)$ & $50.0(31.6-50.0)\left(P=.031^{b}\right)$ & $100(96.1-100)(\mathrm{NA})$ & $100(63.2-100)$ & $90.4(86.8-90.4)$ \\
\hline Superficial lobe & 8 & 75 & & & & & \\
\hline \multicolumn{8}{|l|}{ 4) UL } \\
\hline Deep lobe & 8 & 8 & $82.4(74.6-89.5)\left(P=.002^{b}\right)$ & $50(27.8-70.1)(P=.070)$ & $89.3(84.6-93.6)\left(P=.008^{b}\right)$ & $50(27.8-70.1)$ & $89.3(84.6-93.6)$ \\
\hline Superficial lobe & 8 & 67 & & & & & \\
\hline
\end{tabular}

Note:-PPV indicates positive predictive value; NPV, negative predictive value; NA, not available.

${ }^{a}$ Statistical analyses ( $P$ values) were performed comparing the direct method with each indirect method

${ }^{\mathrm{b}}$ Significant difference $(P<.05)$.

there have been no previous studies evaluating the 3D-DESS-WE sequence for localizing parotid gland tumors.

In this study, the direct method using the 3D-DESS-WE sequence showed higher accuracy, sensitivity, specificity, positive predictive value, and negative predictive value than indirect methods. Therefore, we found that the 3D-DESS-WE sequence could reliably and accurately depict the intraparotid facial nerve in the presence of parotid gland tumors. However, 2 cases were incorrectly categorized. One lesion was incorrectly categorized to the superficial lobe because a linear high-intensity structure was seen to course medial to the parotid gland tumor, but surgical findings confirmed the presence of a thin branch of the intraparotid facial nerve lying on the surface of the parotid gland tumor, therefore categorizing this as a deep lobe lesion. In another case, the tumor was located in the far distal (anterior) part of parotid gland parenchyma, rendering visualization of the intraparotid facial nerve limited on the 3D-DESS-WE sequence and therefore limiting our ability to localize this lesion. In this case, we categorized the lesion to the superficial lobe based on a plane parallel to the visualized facial nerve trunk. However, surgical findings confirmed the parotid gland tumor localized to the deep lobe.

As mentioned in the Results section, 6 of 91 lesions had to be localized using this method, and this was the only case found to be incorrect. Moreover, this highlights the difficulty in visualizing the intraparotid facial nerve in the far anterior part of the parotid gland because the distal branches of the facial nerve become increasingly thin. However, an isolated injury to a thin, peripheral branch of the facial nerve is less likely to have significant consequences. Nonetheless, our study demonstrated that we could achieve high diagnostic performance in parotid tumor localization using the 3D-DESS-WE sequence with a standard, commercially available head and neck coil. This study showed excellent interobserver variability of 3D-DESS-WE, which was higher than that of the indirect methods. Similarly, a previous study showed that the 3D-DESS-WE sequence enabled uniform detectability of the peripheral branches of the cranial nerve by readers at various training levels. ${ }^{10}$

There are several limitations in this study. First, our study consists of a relatively small number of deep lobe tumors (17.6\%) compared with superficial lobe tumors. However, it is wellknown that the occurrence of parotid tumors in the deep lobe is approximately $10 \%$, which was concordant with this study. ${ }^{19}$ Furthermore, the number of deep lobe tumors was sufficient to show statistical significance in this study. A second limitation was the disuse of a surface coil, which could achieve better visualization of the intraparotid facial nerve. ${ }^{20,21}$ However, we found that using the surface coil was not feasible in routine clinical practice due to its time-consuming and cumbersome application. Despite the lack of a surface coil, this study still demonstrated good diagnostic performance using a standard, commercially available head and neck coil, and the acquisition time of the 3D-DESS-WE sequence was relatively short, maintaining its feasibility for routine clinical practice. A third limitation was a small number of nonfatty parotid glands, which could negatively influence the diagnostic performance. In a patient with a nonfatty parotid gland, it is difficult to trace the course of the intraparotid facial nerve because of low contrast between the nerve and parotid parenchyma. Generally, older patients have more fatty infiltration of the parotid gland. A relatively high median age of the patients was thought to explain the small number of nonfatty parotid glands in this study.

\section{CONCLUSIONS}

Direct visualization of the intraparotid facial nerve using the 3DDESS-WE sequence demonstrated better diagnostic performance in localizing parotid gland tumors compared with various indirect methods. This technique can be performed without a surface coil and with a relatively short acquisition time and has high interobserver variability. These features make the 3D-DESS-WE sequence feasible for routine clinical practice and could be an integral part of preoperative assessment of parotid gland tumors.

Disclosures: Osamu Sakai-UNRELATED: Consultancy: Boston Imaging Core Lab; Royalties: Gakken Medical Shujunsha Co Ltd, Medical Sciences International Ltd.

\section{REFERENCES}

1. Teresi LM, Kolin E, Lufkin RB, et al. MR imaging of the intraparotid facial nerve: normal anatomy and pathology. AJR Am J Roentgenol 1987;148:995-1000 CrossRef Medline

2. Takahashi N, Okamoto K, Ohkubo M, et al. High-resolution magnetic resonance of the extracranial facial nerve and parotid duct: demonstration of the branches of the intraparotid facial nerve and its relation to parotid tumours by MRI with a surface coil. Clin Radiol 2005;60:349-54 CrossRef Medline 
3. de Ru JA, van Benthem PP, Hordijk GJ. The location of parotid gland tumors in relation to the facial nerve on magnetic resonance images and computed tomography scans. J Oral Maxillofac Surg 2002;60: 992-94; discussion 995 CrossRef Medline

4. Ariyoshi Y, Shimahara M. Determining whether a parotid tumor is in the superficial or deep lobe using magnetic resonance imaging. J Oral Maxillofac Surg 1998;56:23-26; discussion 26-27 CrossRef Medline

5. Divi V, Fatt MA, Teknos TN, et al. Use of cross-sectional imaging in predicting surgical location of parotid neoplasms. J Comput Assist Tomogr 2005;29:315-19 CrossRef Medline

6. Kurabayashi T, Ida M, Ohbayashi N, et al. Criteria for differentiating superficial from deep lobe tumours of the parotid gland by computed tomography. Dentomaxillofac Radiol 1993;22:81-85 CrossRef Medline

7. Ishibashi M, Fujii S, Kawamoto K, et al. The ability to identify the intraparotid facial nerve for locating parotid gland lesions in comparison to other indirect landmark methods: evaluation by $3.0 \mathrm{~T}$ MR imaging with surface coils. Neuroradiology 2010;52:1037-45 CrossRef Medline

8. Li C, Li Y, Zhang D, et al. 3D-FIESTA MRI at $3 \mathrm{~T}$ demonstrating branches of the intraparotid facial nerve, parotid ducts and relation with benign parotid tumours. Clin Radiol 2012;67:1078-82 CrossRef Medline

9. Tsang JC, Yip WH, Lau CS, et al. Visualization of normal intraparotid facial nerve on MR: BTFE or GRASS? Clin Radiol 2009;64: 1115-18 CrossRef Medline

10. Fujii H, Fujita A, Yang A, et al. Visualization of the peripheral branches of the mandibular division of the trigeminal nerve on $3 \mathrm{D}$ double-echo steady-state with water excitation sequence. $A J N R$ Am J Neuroradiol 2015;36:1333-37 CrossRef Medline

11. Qin Y, Zhang J, Li P, et al. 3D double-echo steady-state with water excitation MR imaging of the intraparotid facial nerve at 1.5T: a pilot study. AJNR Am J Neuroradiol 2011;32:1167-72 CrossRef Medline
12. Ruohoalho J, Mäkitie AA, Aro K, et al. Complications after surgery for benign parotid gland neoplasms: a prospective cohort study. Head Neck 2017;39:170-76 CrossRef Medline

13. Eisele DW, Wang SJ, Orloff LA. Electrophysiologic facial nerve monitoring during parotidectomy. Head Neck 2010;32:399-405 CrossRef Medline

14. Lydiatt DD. Medical malpractice and facial nerve paralysis. Arch Otolaryngol Head Neck Surg 2003;129:50-53 CrossRef Medline

15. Ryzenman JM, Pensak ML, Tew JM Jr. Facial paralysis and surgical rehabilitation: a quality of life analysis in a cohort of 1,595 patients after acoustic neuroma surgery. Otol Neurotol 2005;26:516-21; discussion 21 CrossRef Medline

16. Kim JY, Yang HC, Lee S, et al. Effectiveness of anatomic criteria for predicting parotid tumour location. Clin Otolaryngol 2016;41: 154-59 CrossRef Medline

17. Imaizumi A, Kuribayashi A, Okochi K, et al. Differentiation between superficial and deep lobe parotid tumors by magnetic resonance imaging: usefulness of the parotid duct criterion. Acta Radiol 2009; 50:806-11 CrossRef Medline

18. Zhang Z, Meng Q, Chen Y, et al. 3-T imaging of the cranial nerves using three-dimensional reversed FISP with diffusion-weighted MR sequence. J Magn Reson Imaging 2008;27:454-58 CrossRef Medline

19. Harney MS, Murphy C, Hone S, et al. A histological comparison of deep and superficial lobe pleomorphic adenomas of the parotid gland. Head Neck 2003;25:649-53 CrossRef Medline

20. Chu J, Zhou Z, Hong G, et al. High-resolution MRI of the intraparotid facial nerve based on a microsurface coil and a $3 \mathrm{D}$ reversed fast imaging with steady-state precession DWI sequence at $3 \mathrm{~T}$. AJNR Am J Neuroradiol 2013;34:1643-48 CrossRef Medline

21. Zhao Y, Yang B. Value of visualization of the intraparotid facial nerve and parotid duct using a micro surface coil and three-dimensional reversed fast imaging with steady-state precession and diffusion-weighted imaging sequence. J Craniofac Surg 2018;29:e754-57 CrossRef Medline 